\title{
A Multicenter, Open-Label Study of the Efficacy and Safety of Telmisartan in Mild to Moderate Hypertensive Patients
}

\author{
Frida Liane Plavnik and Artur Beltrame Ribeiro (on behalf of the Brazilian Group of \\ Telmisartan Investigators *)
}

São Paulo, SP

\begin{abstract}
Objective - To evaluate the efficacy and tolerability of telmisartan, given once a day to patients with mild to moderate hypertension, as well as to assess the 24-hour blood pressure profile with ABPM.

Methods - Initially, 163 patients over 18 were selected, regardless of sex, with blood pressure levels $>140 / 90 \mathrm{mmHg}$ at visit 1 , which was confirmed at visit 2 . One hundred thirty-four patients completed the study. After a 4-week placebo run-in phase, telmisartan $40 \mathrm{mg} /$ daily was given for 6 weeks. In those patients whose blood pressure (BP) levels were lower than 140/90mmHg, the same dosage was kept for an additional period of 6 weeks. For those who had BP higher than $140 / 90 \mathrm{mmHg}$, the dosage was increased to $80 \mathrm{mg} /$ daily. Sixty-two patients were included in a subgroup that underwent ABPM 3 different times during the study.
\end{abstract}

Results - In the overall group, blood pressure reduction ranged from $162.3 \pm 14.5 / 101.3 \pm 5.75 \mathrm{mmHg}$ (baseline) to $147.3 \pm 20.1 / 90.8 \pm 10.9 \mathrm{mmHg}$ (week 12) $(p<0.05)$. Mean blood pressure decreases were $14.4 \mathrm{mmHg}$ for systolic $B P$ and $10.3 \mathrm{mmHg}$ for diastolic BP, after 12 weeks of active treatment. A subanalysis showed that 47 (35\%) patients took telmisartan 40mg throughout the study and 81 (65\%) had the dosage increased to 80mg daily. Using ABPM, an 8$\mathrm{mmHg}$ reduction in systolic $B P$ as well as a $5-\mathrm{mmHg}$ reduction in diastolic $B P$ were observed, when compared with baseline values in the final 6 hours (18-24 hours after the last dose of study medication).

Conclusion - Our results confirm that telmisartan given once a day is effective in reducing blood pressure levels in mild to moderate hypertensive patients. This reduction occurs in a sustained and gradual manner during a 24-hour period confirmed by $A B P M$.

Key-words: telmisartan, hypertension, ABPM

Mailing address: Frida Liane Plavnik - Hospital do Rim e Hipertensão - Fundação Osvaldo Ramos - Rua Borges Lagoa, 960 - 04038-002

E-mail: flpavnik@zaz.com.br
The ideal antihypertensive agent must have the following profile: it must promote effective blood pressure (BP) reduction, maintain blood pressure control over 24 hours with a single daily dose, be as effective as possible for a great number of hypertensive patients, cause no undesirable side effects have no negative effects on metabolic parameters ${ }^{1}$, and finally be reasonably affordable. None of the antihypertensive agents currently available have all of these characteristics. More recently, some new drugs have demonstrated some of the desired characteristics of an ideal drug.

The maintenance of blood pressure reduction during the first hours of the morning, after the patient wakes up, is another major point to be considered, because the most likely peak time for cardiovascular events occurs between 06:00-11:00 2-5. The goals for blood pressure levels have become increasingly rigorous. The new goals are based on the results from the most recent prospective clinical studies, such as HOT ${ }^{6}$ and UKPDS ${ }^{7}$, which demonstrate that more rigorous blood pressure control provides greater protection against cardiovascular morbidity and mortality in hypertensive populations. These findings emphasize that antihypertensive agents must keep blood pressure under control for the longest period of time possible.

The renin-angiotensin system (RAS) plays an important role in the pathogenesis and maintenance of the mechanisms involved in the development of essential hypertension. Some of its actions have been well characterized ${ }^{8}$. Briefly, angiotensin II (AII) acts by binding to its specific subtype $\mathrm{AT}_{1}$ receptor, leading to vasoconstriction, increasing peripheral resistance (raising blood pressure levels), and promoting some trophic actions (wall and heart vessels hypertrophy).

A new class of antihypertensive agents, the angiotensin II (AII) antagonist receptors (AIIAR) (ie, losartan, valsartan, irbesartan, candesartan, and telmisartan), blocks the binding of AII to its subclass receptor $\mathrm{AT}_{1}$. This action prevents the potent vasoconstriction generated by AII. 
Although different drugs of this class have a similar pharmacokinetics and pharmacodynamic profile, it has been shown that telmisartan seems to have some special characteristics, such as a longer half-life (24-hour elimination) and an "insurmountable" antagonism to the $\mathrm{AT}_{1}$ receptor in a concentration-dependent manner ${ }^{9}$. It means that telmisartan has a slower dissociation rate from its receptor, providing greater and longer stability. AIIARs have an excellent tolerability profile, and telmisartan has a tolerability profile at least similar to that observed with placebo ${ }^{9}$.

The aim of this study was to evaluate the efficacy and tolerability of telmisartan given once daily to patients with mild to moderate hypertension, as well as to assess the 24hour blood pressure profile by using ambulatory blood pressure monitoring (ABPM).

\section{Methods}

Patients with blood pressure levels of $140 / 90 \mathrm{mmHg}$ or higher (confirmed on 2 consecutive visits), who were 18 years old and older, regardless of sex, were selected. The exclusion criteria were as follows:

Suspected or known diagnosis of arterial hypertension with a secondary cause; women who were not using any effective method of contraception, or who were pregnant, or breast-feeding; systolic blood pressure $>200 \mathrm{mmHg}$ or diastolic blood pressure $>115 \mathrm{mmHg}$; laboratory test values twofold above the upper normal limit; previous intolerance to angiotensin conversion enzyme inhibitors or AIIAR; New York Heart Association class III or IV heart failure; history of stroke in the 6 months prior to entry into the study; uncontrolled type 2 diabetes mellitus, defined as fasting glucose levels $>140 \mathrm{mg} / \mathrm{dL}$ on 3 consecutive assessments; patients who were included in another investigational drug study in the past 30 days.

At the initial evaluation (visit 1), each patient received a detailed explanation of the objectives and procedures of this study. They were asked to sign an informed consent to participate before any procedure was done. All patients had a history of previous treatment for high blood pressure.

This protocol was submitted to and approved by the Ethics Committee of each participating center.

This was an open, phase IV, prospective, run-in with placebo, 12-week active treatment, multicenter study (14 sites). Figure 1 shows the recruitment algorithm and the number of patients screened in this study.

The study consisted of a placebo run-in period in which the placebo was given in the morning for 4 weeks to confirm blood pressure levels and to allow assessment of treatment compliance. That was followed by a 6 -week period when all patients received 1 tablet of telmisartan $40 \mathrm{mg}$ once a day in the morning. At the end of this period, patients whose ambulatory blood pressure levels were below 140/ $90 \mathrm{mmHg}$ were asked to continue taking $40 \mathrm{mg}$ for an additional period of 6 weeks. Patients whose blood pressure was higher than 140/90mmHg had their telmisartan dosage increased to $80 \mathrm{mg} /$ day, 2 tablets in the morning.

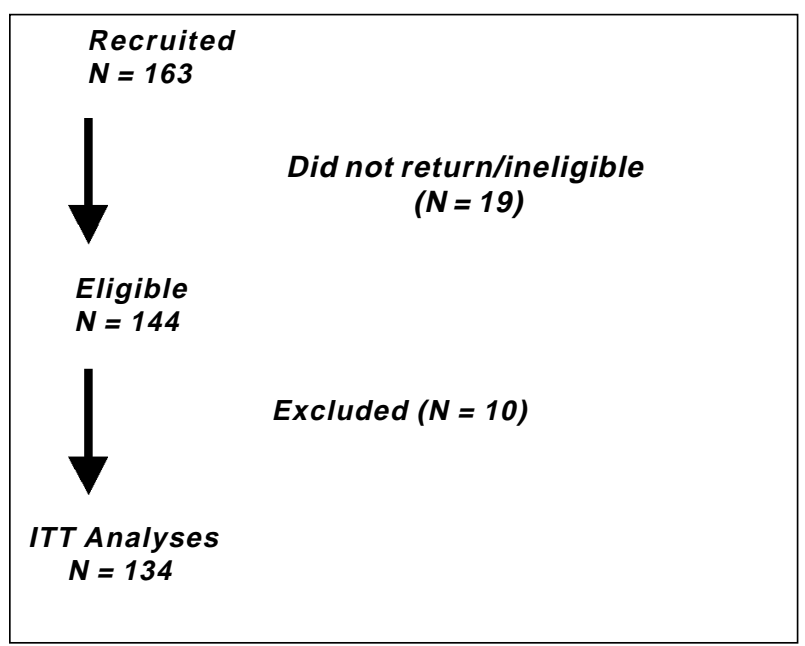

Fig. 1 - Algorithm for patient selection; ITT, intention to treat.

Sixty-two (50\%) patients underwent ambulatory blood pressure monitoring (ABPM) on 3 different occasions: at the end of visits 2,4 , and 6 , which correspond to the end of the placebo phase, the telmisartan 40-mg phase, and the end of the study, when they received 40 or $80 \mathrm{mg}$ of telmisartan, respectively.

Laboratory safety assessments were performed at the end of the run-in period and at the end of the study. The assessed parameters were hematology (count of red blood cells, white blood cells, differential and platelets), creatinine, sodium, potassium, glucose, AST/SGOT (serum glutamic-oxaloacetic transaminase), ALT/SGPT (serum glutamicpyruvic transaminase), and ECG (electrocardiogram).

The primary efficacy variables were SBP/DBP variation, obtained 24 hours after the last dose (through values) at the office, after 6 and 12 weeks, from a baseline measurement taken with the patient seated.

The secondary efficacy variables were response percentile (DBP $<90 \mathrm{mmHg}$ and $/$ or decrease $\geq 10 \mathrm{mmHg}$; SBP $<140 \mathrm{mmHg}$ and/or a reduction $>10 \mathrm{mmHg}$ ) and blood pressure values changes on ABPM after 6 and 12 weeks of treatment. The safety evaluation was based on the reports of clinical adverse events or laboratory findings. Figure 2 shows the study design.

Office blood pressure was measured according to American Heart Association guidelines ${ }^{10}$. Blood pressure measurement was performed 3 times with the patient seated, after a 5-minute rest. The average was used for the subsequent data analysis. A heart rate was also taken (measured for 30 seconds and expressed as beats per minute).

Ambulatory blood pressure monitoring was performed with an automated device (SpaceLabs model 90207). The measurements were transmitted to the analysis system (SpaceLabs model 90209, Data Interface Unit), which performs the measurements with an oscillating method. The period of 24-hour clock time was divided into day vigil (the time between waking up and going to sleep) and night sleep (the time between the moment that the patient falls asleep until the moment he wakes up). During the vigil period, BP 
measurements were taken every 15 minutes (4/hour), and during the sleeping period, the measurements were taken every 20 minutes (3/hour). The monitor was installed in the morning, turned on between 08:00 AM - 10:00 AM, always before the daily medication dose was taken. The appraised parameters were the 24-hour SBP/DBP average, the SBP/ DBP average for the vigil and sleeping periods. The examination was considered valid when more than $80 \%$ of the readings were available. In the case of measurement flaws for 3 consecutive hours or 6 nonconsecutive hours, the examination was repeated.

The data are shown as average and standard deviation and, when appropriate, as a function of the sample distribution characteristics, for the median and its confidence intervals. The database was stored and analyzed with Sigma Stat 1.0 software (Chicago, IL, 1991). The following statistical tests were used: paired t test (ABPM measurements), ANOVA (comparison of the clinical BP measurements in the whole group). The telmisartan 40-mg and 80-mg subgroups were analyzed with the nonpaired $t$ test or the Mann-Whitney test, when the sample distribution homogeneity was not seen. It was considered significant a $\mathrm{p}<0.05$.

\section{Results}

As shown in figure 1,163 subjects were initially recruited. One hundred thirty- four of those recruited patients were included in the study. Twenty-nine patients were excluded from the study: 19 did not return for their scheduled appointments (visit 2) or were not eligible, and 10 patients were excluded due to loss to follow-up, withdrawal of consent, or blood pressure levels outside the range specified in the protocol. A subanalysis of the whole group showed that $47(35 \%)$ patients received telmisartan $40 \mathrm{mg}$ for 12 weeks, and $81(65 \%)$ patients had their dosage increased to $80 \mathrm{mg}$.

The mean age was $57 \pm 10$ years old (ranging from 32 80 years). The group had 93 females and 41 males. With regard to ethnic background, $54.4 \%$ were white, $23 \%$ mulatto, $21 \%$ black, and $1.5 \%$ Asian or unknown. The time between the hypertension diagnosis and the beginning of the study was 10.7 (3.9-18.9) years. Sixty-seven percent of the patients never smoked, $24.6 \%$ were former smokers, and $8.4 \%$ were smokers. With regard to alcohol consumption, $89.5 \%$ never consumed any type of alcoholic drink and 10.5\% consumed alcohol sporadically.

Table I shows the data of the 2 dosage subgroups. No statistically significant difference was observed between demographic characteristics, except for the time of the diagnosis of hypertension. This subgroup $(n=47)$ reported a more recent diagnosis than the group that received $80 \mathrm{mg}$.

Table II shows the $\mathrm{BP}$ values and heart rate averages taken after placebo use (V2), 6 weeks after telmisartan $40 \mathrm{mg}$ use (V4), and at the end of the study (V6) for the entire group. No differences were observed in heart rate at any point. The BP level differences are shown elsewhere. Figure 3 shows BP reductions observed after 6 and 12 weeks of

\begin{tabular}{|c|c|c|}
\hline Group/parameter & $\begin{array}{c}\mathrm{T} 40 \\
(\mathrm{n}=47)\end{array}$ & $\begin{array}{c}\mathrm{T} 80 \\
(\mathrm{n}=81)\end{array}$ \\
\hline Age (years) & $57 \pm 10$ & $57 \pm 10$ \\
\hline \multicolumn{3}{|l|}{ Sex } \\
\hline Female/male & $34 / 13$ & $55 / 26$ \\
\hline \multicolumn{3}{|l|}{ Race } \\
\hline White & 31 & 40 \\
\hline Black & 9 & 17 \\
\hline Mulatto & 7 & 22 \\
\hline Other & 0 & 1 \\
\hline Unknown & 0 & 1 \\
\hline Height $(\mathrm{cm})$ & $160.5 \pm 8.3$ & $159.1 \pm 8.4$ \\
\hline \multicolumn{3}{|l|}{ Weight (kg) } \\
\hline V2 & $71 \pm 14$ & $71 \pm 12$ \\
\hline BMI $\left(\mathrm{kg} / \mathrm{m}^{2}\right)$ & $27 \pm 4$ & $28 \pm 4$ \\
\hline \multicolumn{3}{|l|}{ Smoking } \\
\hline Current & 6 & 4 \\
\hline Former-smoker & 12 & 21 \\
\hline Never smoked & 29 & 56 \\
\hline \multicolumn{3}{|l|}{ Alcohol consumption } \\
\hline Yes & 4 & 9 \\
\hline No & 43 & 72 \\
\hline Time of diagnosis (years) & $\begin{array}{c}5.3 \\
(2.00-128)\end{array}$ & $\begin{array}{c}13.8 \\
(5.88-21.0) *\end{array}$ \\
\hline \multicolumn{3}{|c|}{$\begin{array}{l}\text { * } \mathrm{P}=0.001 ; \mathrm{T} 40 \mathrm{mg} \text { vs. T } 80 \mathrm{mg} \text {. (Mann-Whitney test). T } 40=\text { patients } \\
\text { receiving telmisartan } 40 \mathrm{mg} \text { throughout the study; T } 80=\text { patients who } \\
\text { had their dose increased to } 80 \mathrm{mg} \text { after } 6 \text { weeks of treatment with } \\
\text { telmisartan } 40 \mathrm{mg} \text { daily due to poor blood pressure control. }\end{array}$} \\
\hline
\end{tabular}

\begin{tabular}{|lccc|}
\hline \multicolumn{4}{|c|}{$\begin{array}{c}\text { Table II - Mean and standard deviation of blood pressure and } \\
\text { heart rate values obtained in the office }(\mathbf{n}=\mathbf{1 3 4})\end{array}$} \\
\hline Group/parameter & $\mathrm{V} 2$ & $\mathrm{~V} 4$ & $\mathrm{~V} 6$ \\
\hline SBP $(\mathrm{mmHg})$ & $162 \pm 14$ & $152 \pm 22$ & $147 \pm 20$ \\
DBP $(\mathrm{mmHg})$ & $101 \pm 6$ & $94 \pm 12$ & $91 \pm 11$ \\
HR $(\mathrm{bpm})$ & $73 \pm 9$ & $73 \pm 9$ & $72 \pm 8$ \\
\hline \multicolumn{2}{l}{ SBP- systolic blood pressure; DBP- diastolic blood pressure; } \\
HR- heart rate.
\end{tabular}

treatment with telmisartan. Those patients requiring $80 \mathrm{mg}$ of the study drug showed a statistically significant BP level reductions after 6 weeks of treatment, which persisted after doubling the dosage.

The average BP reduction was $14.4 \mathrm{mmHg}$ for systolic $\mathrm{BP}$ and $10.3 \mathrm{mmHg}$ for the diastolic BP after the 12-week treatment.

Sixty-two patients participated in ABPM. A statistically significant $\mathrm{BP}$ reduction was seen after the drug treatment compared with that at the end of placebo period, both after 6 and 12 weeks of treatment, as shown in figures 4 to 7 .

According to the ABPM data, a reduction of $8 \mathrm{mmHg}$ in SBP and 5mmHg in DBP was observed in the last 6 hours recorded, after 6 weeks of therapy (18-24 hours after drug intake), compared with baseline values. In addition, the ABPM performed after 12 weeks, following the beginning of treatment showed 5-mmHg and 4-mmHg reductions, respectively ( $\mathrm{p}<0.05$ vs. placebo).

Regarding the response percentiles, a reduction in the 


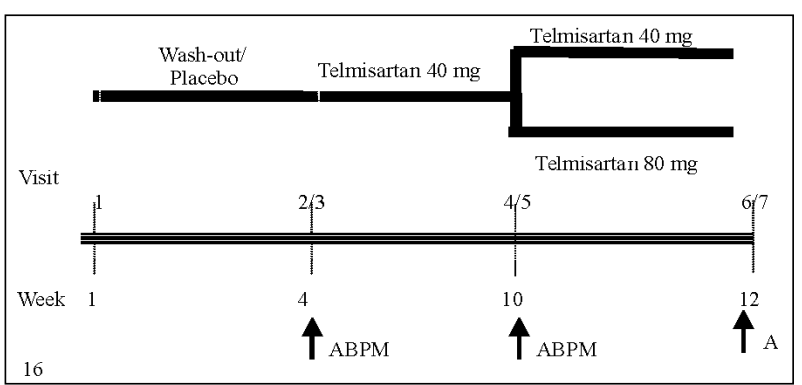

Fig. 2 - Study design.

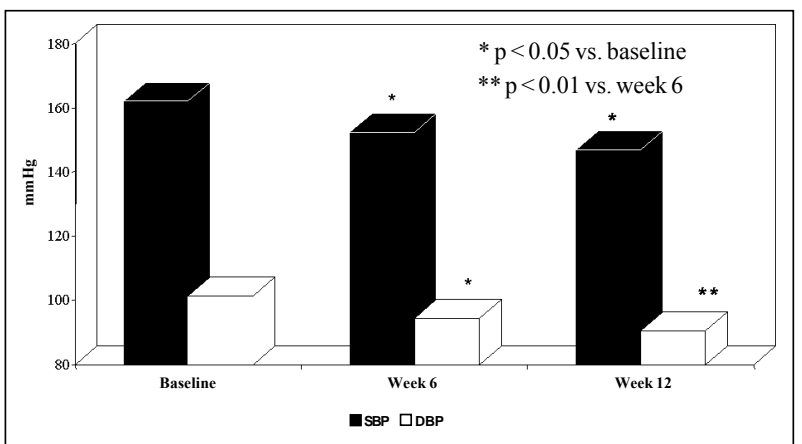

Fig. 3 - Effect of telmisartan on systolic and diastolic blood pressure levels after 6 and 12 weeks of treatment $(n=134)$.

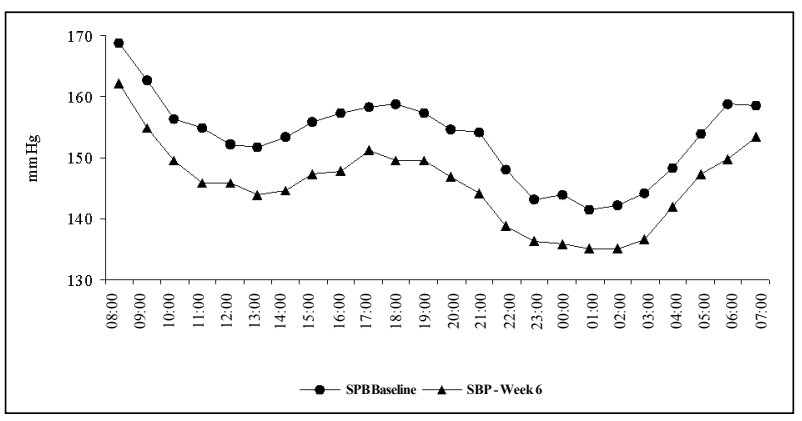

Fig. 4 - Hourly average values at ABPM - systolic blood pressure (baseline vs. week 6).

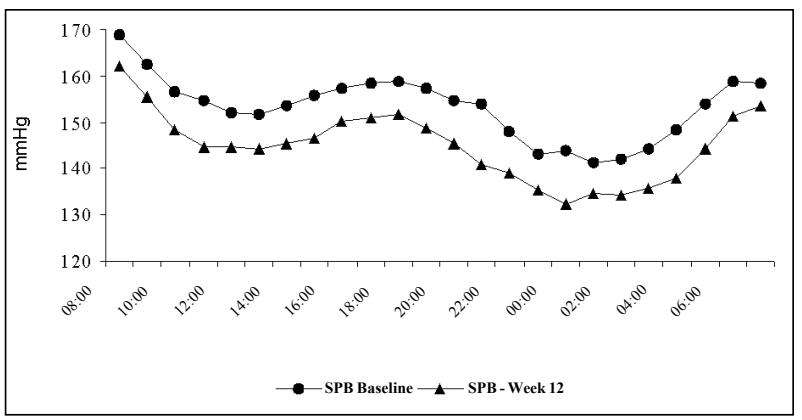

Fig. 5 - Hourly average values at ABPM - systolic blood pressure (baseline vs. week 12).

SBP levels $<140 \mathrm{mmHg}$ (around $10 \mathrm{mmHg}$ in $63 \%$ of the patients), whiletheDBPreduction to $90 \mathrm{mmHg}$ or lower $(10 \mathrm{mmHg}$ in $57.5 \%$ of the patients). BP control (DBP $<90 \mathrm{mmHg}$ ) was reached by $52 \%$ of the patients.

Sixty-nine patients (51\%) reported 118 clinical adverse

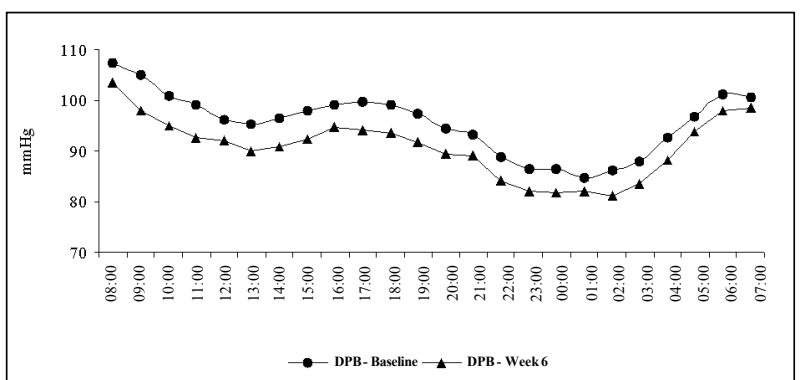

Fig. 6 - Hourly average values at ABPM - diastolic blood pressure (baseline vs. week 6).

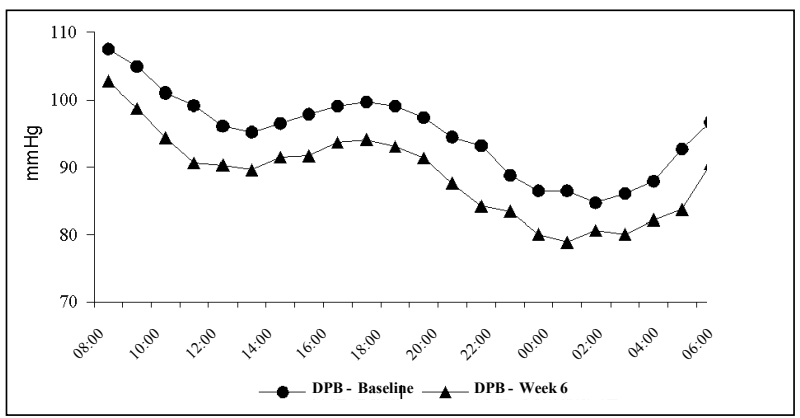

Fig. 7 - Hourly average values at ABPM - diastolic blood pressure (baseline vs. week 12).

events. Most of them (72.4\%) were considered mild. Two serious adverse events not related to the study medication were reported: a case of bronchopneumonia and a stroke in a patient who discontinued the medication independently at the beginning of the study.

The events more commonly reported (incidence $>1 \%$ ) were migraine, gastric pain, throat infections, and palpitation (at the end of the placebo phase). Other adverse events, such as migraine, nasal bleeding, and upper airways infection, were reported after 6 weeks of treatment. Migraine, dizziness, bronchitis, and back pain were reported at the end of the study.

Migraine was reported mostly during the placebo period $(13.5 \%$ in the placebo period versus $9 \%$ in the active treatment period; $p<0.05$ vs. placebo phase). Other clinical adverse events considered not serious had no significant differences.

Table III shows the laboratory evaluations before and after treatment. No significant or clinical changes were observed in blood count, and metabolic, hepatic, and renal function tests after telmisartan treatment.

Significant alterations were not observed in the ECG pattern before and after treatment with telmisartan .

\section{Discussion}

The choice of $40 \mathrm{mg}$ and $80 \mathrm{mg}$ doses of telmisartan used in this study is based on previous studies evaluating the efficacy of different dosages (range: $20-160 \mathrm{mg}$ daily) on blood pressure reduction ${ }^{9,11,12}$. In these studies, the authors observed that $40 \mathrm{mg}$ of telmisartan provides significant de- 


\begin{tabular}{|lcc|}
\hline \multicolumn{3}{|c|}{$\begin{array}{c}\text { Table III - Laboratory tests at the end of the placebo period and } \\
\text { after 12 weeks of treatment with telmisartan }\end{array}$} \\
\hline Parameter & Baseline & $\mathrm{V} 6$ \\
\hline Sodium $(\mathrm{mEq} / \mathrm{L})$ & $140 \pm 3$ & $140 \pm 3$ \\
Potassium $(\mathrm{mEq} / \mathrm{L})$ & $4.2 \pm 0.5$ & $4.3 \pm 0.7$ \\
Creatinine $(\mathrm{mg} / \mathrm{dL})$ & $0.8 \pm 0.2$ & $0.9 \pm 0.2 *$ \\
Glucose $(\mathrm{mg} / \mathrm{dL})$ & $98 \pm 18$ & $98 \pm 23$ \\
ALT $(\mathrm{UI} / \mathrm{L})$ & $21 \pm 8$ & $20 \pm 7$ \\
AST $(\mathrm{UI} / \mathrm{L})$ & $19 \pm 9$ & $19 \pm 8$ \\
\hline \multirow{2}{*}{$* p=0.006$ vs. baseline creatinine; AST/SGOT- serum glutamic-oxaloa- } \\
cetic transaminase ALT/SGPT- serum glutamic-pyruvic transaminase. \\
\hline
\end{tabular}

creases in blood pressure, from 9-11 $\mathrm{mmHg}$ and $6-8 \mathrm{mmHg}$, respectively, for systolic and diastolic BP, and increasing the dosage to $80 \mathrm{mg}$ resulted in a greater antihypertensive response than with the $40 \mathrm{mg}$ dosage. They also observed that dosages of 120-160 mg did not promote further decreases. Therefore, the telmisartan dosages established to obtain an effective reduction in BP levels without impairment of tolerability were 40 and $80 \mathrm{mg} /$ daily.

This study showed that in this group of hypertensive subjects, a mean reduction occurred of $10 / 7 \mathrm{mmHg}$ (SBP and DBP, respectively) after 6 weeks of treatment with telmisartan 40mg, once a day. An additional reduction was observed when the dosage was increased to $80 \mathrm{mg}$ a day $(4.4 / 3.3$ $\mathrm{mmHg}$ for SBP and DBP). An overall reduction of $14.4 / 10.3$ $\mathrm{mmHg}$ (SBP and DBP) was seen in all subjects after 12 weeks of treatment. These data are consistent with data reported by Neutel et al ${ }^{9}$ and Smith et al ${ }^{11}$ who obtained a significant reduction in blood pressure levels with $40 \mathrm{mg}$ and an additional reduction with $80 \mathrm{mg}$.

Analyzing the group of patients who took only $40 \mathrm{mg}$ of telmisartan throughout the study, we observed that a reduction of $22.4 / 16.3 \mathrm{mmHg}$ (SBP and DBP, respectively) occurred after the first 6 weeks of treatment. This decrease was considered consistent and was maintained until the end of the study. For the group of patients whose medication dosage was increased to $80 \mathrm{mg}$, a slight reduction occurred (9.9/7.1 mmHg, with SBP/DBP, respectively). The mean reduction for the entire group is shown in figure 8 . Some authors described similar findings, showing reductions of $11.3 / 7.3 \mathrm{mmHg}$ with telmisartan $40 \mathrm{mg}$ and $13.7 / 8.1 \mathrm{mmHg}$ with telmisartan $80 \mathrm{mg}$ a day compared with placebo ${ }^{9,11}$ and average reductions of 10-11.9 $\mathrm{mmHg}$ for systolic and 8.6-9.7 $\mathrm{mmHg}$ for diastolic BP when different dosages of telmisartan were evaluated ${ }^{12}$. Some studies ${ }^{13}$ have found BP decreases similar in magnitude to those that we observed.

The percentage of patients who achieved a satisfactory response in our study is also consistent with that in preliminary studies ${ }^{9}$. These data show a good response by $51 \%$ for SBP and $43 \%$ for DBP with telmisartan $40 \mathrm{mg}$ once a day, and $57 \%$ and $55 \%$ for SBP and DBP, respectively, with telmisartan $80 \mathrm{mg}$ a day ${ }^{9}$. In general, the percentage of patients with a good response to telmisartan was $63 \%$ for SBP and $57.5 \%$ for DBP. When we analyzed separately those patients who received $40 \mathrm{mg}$ of telmisartan for 12 weeks, we

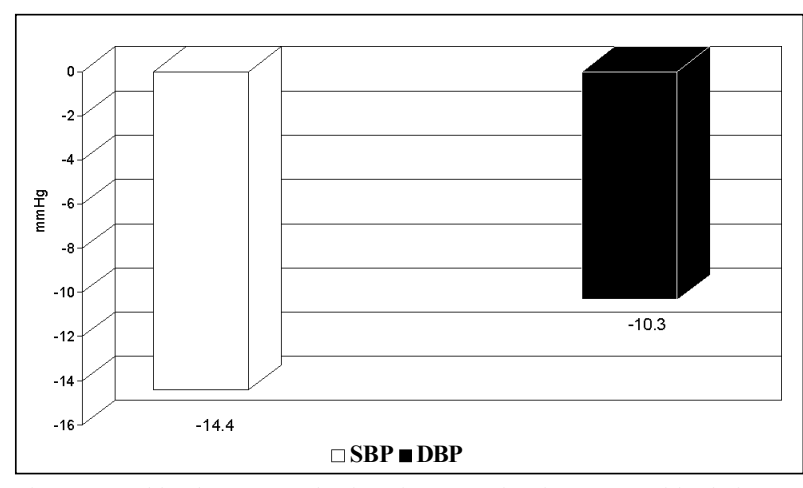

Fig. 8 - Mean blood pressure reduction after 12 weeks of treatment with telmisartan (systolic and diastolic, $\mathrm{N}=134$ ).

noted that $87.2 \%$ had a good response for both systolic and diastolic BP. For the group receiving $80 \mathrm{mg}$, the percentage of responders at the end of the 12 weeks of evaluation was $53.7 \%$ for systolic and $46.2 \%$ for diastolic.

Regarding BP control (DBP $<90 \mathrm{mmHg}$ ), $82 \%$ of the patients who took telmisartan $40 \mathrm{mg}$ had their blood pressure under control, and $33.7 \%$ of those whose medication was raised to $80 \mathrm{mg}$ a day achieved BP control.

A lower percentage of patients achieved BP control with telmisartan $80 \mathrm{mg}$. This finding has been reported by other authors, and it may be related to the fact that a larger number of patients in this group had basal BP levels between $110-114 \mathrm{mmHg}(14.8 \%)$ compared with the group that took telmisartan $40 \mathrm{mg}(2.1 \%)$. Although we could not detect a good response to treatment or control of DBP, we observed a statistically significant reduction in the average $B P$ in this group of patients $(111.7 \pm 1.4$ vs. $101.9 \pm 12.2 p=0.002)$.

No difference was observed with telmisartan and its effect on BP control regarding sex, age, or ethnic background.

Based on ABPM, sustained 24-hour BP control has been shown with telmisartan. Also, this effect on BP persisted during the last 6 hours of the 24-hour BP monitoring and corresponded to that during the 18-24 hours after the ingestion of the last dose of the medication, maintaining BP circadian rhythm, and preventing sudden BP elevation in the first hours of the morning.

Some previous studies have already demonstrated the maintenance of BP control in the last 4 hours with other antihypertensive drugs. It has been shown that telmisartan performs excellently in controlling nocturnal BP when compared with other antihypertensive agents, or even when it is compared with other drugs of its class ${ }^{14-16}$. This long-lasting effect on BP control has been attributed to its longer half-life of approximately 24 hours, with a through to peak ratio greater than $80 \%$. Although these data show that telmisartan maintains its action for 24 hours, we cannot conclude this because we have not compared telmisartan with other antihypertensive agents.

Concerning the tolerance profile, we noted that telmisartan was very well tolerated at both dosages. The incidence of adverse events was smaller during the active treatment phase ( $22 \%$ in the treatment phase with telmisartan 
$40 \mathrm{mg}$, and $29 \%$ in the treatment phase with 40 or $80 \mathrm{mg}$ ) compared with that in the placebo period (39\%). Among all the reported adverse events, migraine was the most common. Its incidence was significantly higher for the placebo period versus the active treatment $(13.5 \%$ vs. 5.1 vs. $3.4 \%, \mathrm{p}<0.05)$.

This excellent tolerability profile seems not to be specific to telmisartan, but an effect of the angiotensin II antagonist class of drugs ${ }^{9,15,17}$. And also, analyzing more than 1400 patients who received this drug versus 380 who took placebo, migraine was the most common adverse event reported. The incidence of adverse events was greater during the placebo period compared with that during the active treatment period ${ }^{18}$.

No significant major laboratory changes could be detected with telmisartan use, which indicates a good laboratory safety profile. The statistical difference that was noted for serum creatinine has no clinical relevance. The mean values before and after treatment remained within the normal range, and, as described for the pharmacokinetics of the drug, renal excretion does not contribute to telmisartan clearance, and no need exists for dosage adjustment in patients with mild to moderate renal impairment. A comparative study between telmisartan (40-80 mg) and enalapril (10-20 mg) in 71 patients with moderate renal impairment showed with renal clearance techniques that telmisartan did not induce any relevant deterioration in renal function. However, in severe renal insufficiency this drug is contraindicated.

Our results confirm that telmisartan, an angiotensin II antagonist, when administered in a daily single dose effectively decreases BP in patients with mild to moderate hypertension and that this decrease is maintained during a 24hour period. ABPM evaluation confirmed that 24-hour BP control, without lapses during the day or increasing BP during the evening, maintains the circadian rhythm pattern. The BP control in the last 6 hours (during a 24-hour control) showed, on average, a persistent reduction compared with baseline values, preventing the sudden BP elevation in the first hours of the morning, which has been associated with cardiovascular events. The tolerability profile was, at least, comparable to that of placebo.

In summary, telmisartan has been shown to be an excellent therapeutic option for treating patients with mild to moderate hypertension, with maintained $\mathrm{BP}$ reduction in office blood pressure values as well as BP measurements using 24-hour ABPM. Its tolerability profile is similar to that of placebo. Telmisartan was effective in preventing sudden BP elevation during the first hours of the morning. It has been shown to be a good option for monotherapy if used in a single daily dose, allowing better patient compliance to pharmacological treatment.

\section{References}

1. Meredith PA, PerloffD, Mancia G, Pickering T. Blood pressure variability and its implications for antihypertensive therapy. Blood Press 1995; 4: 5-11.

2. White WB. Cardiovascular risk and therapeutic intervention for the early morning surge in blood pressure and heart rate. Blood Press Monit 2001; 6: 63-72.

3. Muller JE. Circadian variation and triggering of acute coronary events.Am Heart J 1999; 137(4 Pt 2): S1-S8

4. Sloan MA, Price TR, Foulkes MA, et al. Circadian rhythmicity of stroke onset. Intracerebral and subarachnoid hemorrhage. Stroke 1992; 23: 1420-6.

5. Elliott WJ. Circadian variation in the timing of stroke onset: a meta-analysis. Stroke 1998; 29: 992-6.

6. Hansson L, Zanchetti A, Carruthers SG, et al. Effects of intensive blood-pressure lowering and low-dose aspirin in patients with hypertension. Lancet 1998; 351: 1755-62.

7. UK Prospective Diabetes Study Group. Tight blood pressure control and risk of macrovascular and microvascular complications in type 2 diabetes: UKPDS 38. Br Med J 1998; 317: 703-13.

8. Nicholls MG, Charles CJ, Crozier IG, et al. Blockade of the renin-angiotensin system. J Hypertens 1994; 10(suppl): S95-S103.

9. Neutel JM, Smith DH. Dose response and antihypertensive efficacy of the AT1 receptor antagonist telmisartam in patients with mild to moderate hypertension. Adv Ther 1998; 15: 206-17.

10. PerloffD, Carlene G, Flack J, Frohlich ED, Hill M, McDonald M, Morgenstern BZ. Human blood pressure determination by sphygmomanometry. Circulation 1993; 88 (Part1): 2460-70.
11. Smith DH, Matzek KM, Kempthorne-Rawson J. Dose response and safety of telmisartan in patients with mild to moderate hypertension. J Clin Pharmacol 2000; 40(Pt 1): 1380-90.

12. Smith DHG, Neutel JM, Morgenstern P. Once-daily telmisartan compared with enalapril in the treatment of hypertension. Av Ther 1998; 15: 229-40.

13. Karlberg BE, Lins LE, Hermansson K for the TEES Study Group. Efficacy and safety of tlmisartan, a selective AT1 receptor antagonist, compared with enalapril in elderly patients with primary hypertension. J Hyperten 1999; 17: 293-302.

14. Lacourcière $Y$, Lenis J, Orchard R, et al. A comparison of the efficacies and duration od action of the angiotensin II receptor blockers telmisartan and amlodipine. Blood Press Monit 1998; 3: 295-302.

15. Littlejohn T, Mroczek W, Marbury T, etal. A prospective, randomized, open-label trial comparing telmisartan $80 \mathrm{mg}$ with valsartan $80 \mathrm{mg}$ in patients with mild to moderate hypertension using ambulatory blood pressure monitoring. Can J Cardiol 2000; 16: 1123-32.

16. Mallion J, Siche J, Lacourciere Y. ABPM comparison of the antihypertensive profiles of the selective angiotensin II receptor antagonists telmisartan and losartan in patients with mild-to-moderate hypertension. J Hum Hypertens 1999; 13: 649-50.

17. Neutel JM. Safety and efficacy of angiotensin II receptor antagonists. Am JCardiol 1999; 84: 13K-17K

18. Micardis Product Monograph, 1999 Boehringer Ingelheim Pharmaceuticals Inc, Ridgefield, CT. 